\title{
Care of type II diabetes in older adults: untapped opportunities and remaining challenges
}

Diabetes mellitus type 2 is a chronic metabolic disorder associated with high levels of blood glucose, insulin resistance, and relative lack of insulin. Risk factors for type 2 diabetes include obesity, lack of exercise, and genetic predisposition. About $90 \%$ of cases of diabetes are type 2, and $10 \%$ type 1 and gestational diabetes. If inadequately treated, complications occur almost always, including heart disease, stroke, visual loss, kidney failure, and low blood flow to the limbs leading to infections, gangrene, and amputations. These complications result from macro- and microvascular problems [1]. By contrast, too tight a control can lead to harmful hypoglycemia.

The literature to date reports no significant impact of tight glycemic control on the risk of dialysis/transplantation/renal death, blindness, or neuropathy [2].

According to the American Diabetes Association, one of 3-4 individuals aged $>65$ years has diabetes [3]. Annual screening in older adults permits early detection of individuals at risk for diabetes. Glucose control decreases microvascular events $[1,4-8]$. The effect is proportional to glycated hemoglobin $\left(\mathrm{HbA}_{1 \mathrm{c}}\right)$ reduction and is preserved over time $[4,5]$. Long-term follow-up also shows a benefit on cardiovascular morbidity and mortality $[4,5]$. Delayed intervention may not confer a similar benefit [3-8]. Diabetes may be complicated by chronic infections such as tuberculosis that increase the burden of illness [9].

Early intensive multifactorial intervention can be costeffective in preventing 5-year cardiovascular outcomes in individuals with type 2 diabetes detected by screening [10]. Glycemic and metabolic goals in older adults with diabetes depend on health status, life expectancy, and the number and complexities of complications [11].
Goals directed toward therapies for glucose levels, blood pressure, lipid levels, and lifestyle modifications are targets of interventions. The goals can be modified according to health status, life expectancy, and illness burden [11]. Lifestyles and health status of adults with diabetes such as diet, exercise, smoking, and obesity vary and are heterogeneous. Some are fit and healthy, and others are frail with macro- and microvascular complications that compromise physiological function. Therefore, targets for good control should be individualized. Each patient with diabetes should also receive individualized history taking, physical examination, and counseling. Counseling on nutrition, exercise, smoking cessation, blood pressure control, eye examination, foot, and dental examination are essential periodic routine care. Vaccinations against pneumococcus, influenza, and hepatitis represent some preventive measures [10]. Laboratory monitoring includes periodic fasting lipid profile, $\mathrm{HbA}_{1 \mathrm{c}}$, urine albumin-to-creatinine ratio, and serum creatinine. The appropriate target for $\mathrm{HbA}_{1 \mathrm{c}}$ can also be individualized based on overall health and life expectancy, and identified patient-specific risks for hypoglycemia and the ability of the patient to adopt and adhere to specific treatment regimens $[3-8,10]$. Because overtreatment can result in hypoglycemia, the avoidance of hypoglycemia is an important consideration in establishing goals and choosing therapeutic agents in older adults. In older adults, reducing cardiovascular risk by controlling hypertension and lipid status can also be more useful than tight glycemic control.

Initial therapy in most patients with type 2 diabetes should begin with diet, weight reduction, exercise, and metformin. After a successful initial response, most patients will require additional oral or injectable agents, including insulin

*Correspondence to: Editorial Office of Asian Biomedicine, Faculty of Medicine, Chulalongkorn University, Bangkok 10330, Thailand, e-mail: abmjournal@chula.ac.th

O Open Access. ๑ 2018 Editorial Office of Asian Biomedicine, published by Sciendo. (๔) BY-NC-ND This work is licensed under the Creative Commons Attribution NonCommercial-NoDerivatives 4.0 License. 
for adequate control of blood glucose levels and complications. Clinicians should familiarize themselves with a limited number of insulin regimens to help address patient preferences and lifestyles. The choice of insulin such as basal, prandial, isophane, glargine, or a combination of these insulins has to be targeted toward adequate control based on a combination of $\mathrm{HbA}_{1 \mathrm{c}}$ values and fasting blood glucose levels, diet, and exercise patterns. These should be reviewed periodically by checking glucose levels (fasting, before lunch, before dinner, and before bedtime). Insulin doses can then be adjusted to achieve target levels of glycemia.

\section{References}

[1] Ceriello A, Gavin JR 3rd, Boulton AJM, Blickstead R, McGill M, Raz I, et al. The Berlin declaration: A call to action to improve early actions related to type 2 diabetes. How can specialist care help? Diabetes Res Clin Pract. 2018; 139:392-9.

[2] Rodríguez-Gutiérrez R, Montori VM. Glycemic control for patients with type 2 diabetes mellitus: our evolving faith in the face of evidence. Circ Cardiovasc Qual Outcomes. 2016; 9:504-12.

[3] Lambert M. ADA releases revisions to recommendations for standards of medical care in diabetes. Am Fam Physician. 2012; 85:514-5.

[4] Higgins GT, Khan J, Pearce IA. Glycaemic control and control of risk factors in diabetes patients in an ophthalmology clinic: what lessons have we learned from the UKPDS and DCCT studies? Acta Ophthalmol Scand. 2007; 85:772-6.

[5] Feldman AL, Griffin SJ, Ahern AL, Long GH, Weinehall L, Fhärm $\mathrm{E}$, et al. Impact of weight maintenance and loss on diabetes risk and burden: a population-based study in 33,184 participants. BMC Public Health. 2017; 17:170. doi: 10.1186/s12889-017-4081-6

[6] Hua X, Lung TW, Palmer A, Si L, Herman WH, Clarke P. How consistent is the relationship between improved glucose control and modelled health outcomes for people with type 2 diabetes mellitus? a systematic review. Pharmacoeconomics. 2017; 35:319-29.

[7] Tkáč I. Effect of intensive glycemic control on cardiovascular outcomes and all-cause mortality in type 2 diabetes: overview and
Therefore, proper adherence to medication and injection practices among patients with diabetes cannot be overemphasized to result in optimal health and quality of life [10]. Treatment must be individualized. The article by Dorji et al. in this issue demonstrates that glycemic control and adherence to insulin injection techniques need to be improved in developing countries like Bhutan [12]. The opportunities to improve health and quality of life from good control of diabetes mellitus abound; health providers in the health system and patients have to work hand in hand to overcome the remaining challenges.

metaanalysis of five trials. Diabetes Res Clin Pract. 2009; 86 (Suppl 1):S57-62.

[8] Tandon N, Ali MK, Narayan KM. Pharmacologic prevention of microvascular and macrovascular complications in diabetes mellitus: implications of the results of recent clinical trials in type 2 diabetes. Am J Cardiovasc Drugs. 2012; 12:7-22.

[9] Al-Rifai RH, Pearson F, Crichley JA, Abu-Raddad LJ. Association between diabetes mellitus and active tuberculosis: a systematic review and meta-analysis. PLoS One. 2017; 12:e0187967. doi: 10.1371/ journal.pone.0187967

[10] Simmons RK, Borch-Johnsen K, Lauritzen T, Rutten GEMH, Sandbæk A, van den Donk M, et al. A randomised trial of the effect and cost-effectiveness of early intensive multifactorial therapy on 5 -year cardiovascular outcomes in individuals with screen-detected type 2 diabetes: the Anglo-Danish-Dutch Study of Intensive Treatment in People with Screen-Detected Diabetes in Primary Care (ADDITION-Europe) study. Health Technol Assess. 2016; 20:1-86.

[11] Kirkman MS, Briscoe VJ, Clark N, Florez H, Haas LB, Halter JB, et al. Diabetes in older adults. Diabetes Care. 2012; 35:2650-64.

[12] Dorji T, Lhamo P, Tshering T, Zangmo L, Choden L, Choden D, Namgyal K. Glycemic control, medication adherence, and injection practices among diabetic patients treated in the 3 tertiary referral hospitals in Bhutan: a call for more action. Asian Biomed (Res Rev News). 2018; 12:27-33. 